\title{
On the Solutions of Nonlinear Hybrid Fractional Integrodifferential Equations
}

\author{
Faten H. Damag ${ }^{1}$, Adem Kılıçman ${ }^{2 *}$, Awsan T. Al-Arioi ${ }^{3}$ \\ 1,3 Department of Mathematics, University Taiz, Taiz, Yemen. \\ ${ }^{2}$ Department of Mathematics, University Putra Malaysia, Serdang, Malaysia \\ E-mail: faten_212326@hotmail.com, akilic@upm.edu.my, awsan2018.t@gmail.com
}

\begin{abstract}
In the present work we study the existence of solutions for hybrid nonlinear fractional integrodifferential equations. We developed an algorithm by using the operator theoretical techniques in order to obtain the approximate solutions. The main results depend on the Dhage iteration method that were incorporated with the modern hybrid fixed point theorems. The approximate solutions were obtained by using Lipschitz conditions and weaker form of mixed partial continuity. Further, we provide some examples to explain the hypotheses and the related results.
\end{abstract}

Keywords: Hybrid Fractional Integrodifferential Equation; hybrid fixed point theorems of Dhage; approximations solutions; Lipschitz conditions; weaker mixed partial continuity.

AMS Mathematics Subject Classification: 26A33

\section{Introduction}

Nonlinear fractional integrodifferential equations are important and widely applied in many areas such as in physics, mechanics, electromagnetics, biology, signal processing, economics and more. There are also many different methods to solve these type of equations. In particular, the solutions of existence, uniqueness and other properties for these type of equations have been studied by many authors by using different techniques. In 2016 Dhage et al. (see, [1]) introduced and proved algorithms for the existence of nonlinear first order ordinary integrodifferential equations and the approximation of solutions to initial value problems.In this paper, we extend their study into the classes of nonlinear hybrid fractional integrodifferential equations. Let $J=\left[t_{0}, t_{0}+a\right]$ be a closed-bounded interval in the real space $R$ for some $t_{0}, a \in R$ with $t_{0} \geq 0$ and $a>0$. Consider the nonlinear hybrid fractional integrodifferential equation (Non - HFIDE) of the initial value problem $(I V P)$

$$
\begin{gathered}
\left(D^{\alpha} u\right)(t)+\lambda u(t)=f\left(t, u(t), \int_{t_{0}}^{t} \frac{(t-s)^{\alpha-1}}{\Gamma(\alpha)} g(s, u(s)) d s\right), \\
u\left(t_{0}\right)=B_{0} \in R .
\end{gathered}
$$

where $f: J \times R \times R \rightarrow R$ and $g: J \times R \rightarrow R$ continuous functions and $\lambda \in R(\lambda>0)$, for all $t \in J$. We mean by the solution of Eq.(1), the function $u \in C^{1}(J, R)$ that holds Eq.(1), where $C^{1}(J, R)$ the space of continuously differentiable real-valued functions on $J$.

\section{Preliminaries}

Next, we introduce some related concepts which will be useful in our study, details can be found for example in $[4,5,6,7,8]$ 
Definition 2.1 [2, 3] Let $\alpha \in R_{+}$. The operator $I^{\alpha}$ is defined on $L_{1}[a, b]$ by

$$
I^{\alpha} h(x)=\frac{1}{\Gamma(\alpha)} \int_{a}^{x} \frac{h(\mu)}{(x-\mu)^{1-\alpha}} d \mu, \alpha>0
$$

for $a \leq x \leq b$, is said to be Riemann-Liouville fractional integral having order $\alpha$.

Definition 2.2 [9, 10] Riemann $\&$ Liouville fractional differential operator is defined by

$$
D^{\alpha} h(u)=\frac{1}{\Gamma(m-\alpha)}\left[\frac{d}{d u}\right]^{m} \int_{a}^{u} \frac{h(\mu)}{(u-\mu)^{\alpha-m+1}} d \mu,(m-1) \leq \alpha<m,
$$

where $m$ is an integer and $\alpha$ is a real number.

Throughout this study, $E$ denotes a partially ordered real linear normed space with order relation $\preceq$ and the norm $\|\cdot\|$ in which the scalar product and the addition by non-negative real numbers are preserved by $\preceq$.

The space $E$ is called a regular ( see, [11]) if for any non-increasing (resp., non-decreasing) sequence $\left\{u_{n}\right\}$ in $E$ such that $u_{n} \rightarrow u^{*}$ as $n \rightarrow \infty, u_{n} \succeq u^{*}$ (resp., $u_{n} \preceq u^{*}$ ) for all $n \in N$.

Definition 2.3 [12, 14] A function $T: E \rightarrow E$ is said to be partially continuous at point a $\in E$ if for every $\epsilon>0$ there exists $\delta>0$ such that

$$
\|u-a\|<\delta \Rightarrow\|T u-T a\|<\epsilon
$$

then the map $T$ is called partially continuous on $E$ if it is partially continuous in $E$.

Definition $2.4[13,15]$ A subset $\Omega \neq \emptyset$ of the partially ordered Banach space $E$ is said to be partially compact if $B$ in $\Omega$ is a relatively compact set in $E$.

Definition 2.5 [13, 16] A function $T: E \rightarrow E$ is called:

1. partially compact if $T(E)$ is a partially relatively compact set in $E$,

2. uniformly partially compact if it is partially compact and uniformly partially bounded on E,

3. partially totally bounded if for any bounded subset $\Omega$ of $E, T(\Omega)$ is a partially relatively compact subset of $E$,

4. partially continuous and partially totally bounded, then it is called partially completely continuous on $E$.

Definition 2.6 [12, 1] The metric $d$ and the order relation $\preceq$ on $E \neq \emptyset$ are called compatible if for every monotone sequence $\left\{u_{n}\right\}$ in $E$ with a subsequence $\left\{u_{n k}\right\}$ of $\left\{u_{n}\right\}$ converges to $u^{*}$, the sequence $\left\{u_{n}\right\}$ converges to $u^{*}$

Theorem 2.1 [13] Let $(E, \preceq,\|\|$.$) be a complete regular space partially ordered so that the norm \|\cdot\|$ and the order relation $\preceq$ in $E$ are compatible in any compact chain $C$ of $E$. Then $T: E \rightarrow E$ is a partially continuous, partially compact operator and increasing. If an element $u_{0} \in E$ exists such that $u_{0} \preceq T u_{0}$ or $T u_{0} \preceq u_{0}$, then the equation of the operator $T u=u$ has a solution $u^{*}$ in $E$, and the sequence $\left\{T^{n} u_{0}\right\}$ of successive iterations converges monotonously towards $u^{*}$.

Remark 2.1 The regularity of $E$ in Theorem 2.1 above can be substituted by a stronger continuity condition in the operator $T$ in $E$ (see [13]).

In this work, we will prove the existence and uniqueness solution of the Non - HFIDE (1) by using hybrid fixed point theorems where we need the following notion of a D-function. 
Definition 2.7 An upper semi-continuous and monotone increasing function $\Xi: R_{+} \rightarrow R_{+}$is said to be a D-function provided $\Xi(0)=0$.

Definition 2.8 An operator $T: E \rightarrow E$ is said to be a partial nonlinear $D$-contraction if there is a D-function such that

$$
\|T u-T v\| \leq \Xi(\|u-v\|)
$$

for $u, v \in E$, where $0<\Xi(r)<r, \forall r>0$. In particular, if $\Xi(r)=k r, \forall k>0$, then $T$ is called a partial Lipschitz operator with constant $k$ and moreover, if $0<k<1$, then $T$ is known as partial linear contraction on E.

Theorem $2.2(E, \preceq,\|\|$.$) is a partially ordered, regular, a complete normalized linear space so that$ the norm $\|$.$\| and the order relation \preceq$ in $E$ are compatible in every compact chain $C$ of $E$. Now $A, B: E \rightarrow E$ are two increasing operators such that

(a) the operator $A$ is partially nonlinear D-contraction and partially bounded,

(b) the operator $B$ is partially compact and partially continuous, and

(c) there is an element $u_{0} \in E$ such that $u_{0} \preceq A u_{0}+B u_{0}$ or $u_{0} \succeq A u_{0}+B u_{0}$.

Then the equation $A u+B u=u$ has a solution $u^{*}$ in $E$ and the sequence $\left\{u_{n}\right\}$ of successive iterations introduced by $u_{n+1}=A u_{n}+B u_{n}, \quad n=0,1, \ldots$, converges monotonically to $u^{*}$.

\section{Existence of Solutions and Uniqueness}

The equivalent integral form of Non-HFID (1) is considered in the function space $C(J, R)$ of continuous real-valued functions introduced on $J$. We define the order relation $\preceq$ and a norm $\|\cdot\|$ in $C(J, R)$ by

$$
\|u\|=\sup _{t \in J}|u(t)|
$$

and

$$
u \leq v \Leftrightarrow u(t) \leq v(t), \text { for all, } t \in J
$$

Obviously, $C(J, R)$ is a Banach space with respect to previous supremum norm and is also partially ordered with respect to the previous partially order relation $\preceq$. Further, it is also clear that Banach space of partially ordered $C(J, R)$ is regular and is a lattice, so each pair of elements in the space has an upper and a lower bound in the space.

The following lemma regarding the compatibility of sets in $C(J, R)$ followed by an application theorem of the Arzela-Ascoli.

Lemma 3.3 Let $(C(J, R), \preceq,\|\|$.$) be a Banach space of partially ordered with the order relation \preceq$ and the norm $\|$.$\| introduced by (4) and (5), respectively. Then, \preceq$ and $\|$.$\| are compatible in each partially$ compact subset of $C(J, R)$.

We can see the proof of the lemma in [1]. Before to show our result, we need the following definition:

Definition 3.9 A differentiable function $z \in C^{1}(J, R)$ is a lower solution of the Non - HFIDE (1) if it fulfills

$$
\begin{aligned}
\left(D^{\alpha} z\right)(t)+\lambda z(t) & \leq f\left(t, z(t), \int_{t_{0}}^{t} \frac{(t-s)^{\alpha-1}}{\Gamma(\alpha)} g(s, z(s))\right) d s \\
z\left(t_{0}\right) & \leq B_{0} \in R
\end{aligned}
$$

for every $t$ in J. Likewise, an upper solution $y \in C^{1}(J, R)$ to the Non-HFIDE(1) is introduced on $J$ by inverting the previous inequalities. 
Definition 3.10 A function $z \in C^{1}(J, R)$ is called a lower solution of the Non - HFIDE (19) if it fulfills

$$
\begin{gathered}
\left(D^{\alpha} z\right)(t)+\lambda u(t) \leq f_{1}\left(t, z(t), \int_{t_{0}}^{t} \frac{(t-s)^{\alpha-1}}{\Gamma(\alpha)} g(s, z(s)) d s\right) \\
+f_{2}\left(t, z(t), \int_{t_{0}}^{t} \frac{(t-s)^{\alpha-1}}{\Gamma(\alpha)} g(s, z(s)) d s\right), \\
z\left(t_{0}\right) \leq B_{0},
\end{gathered}
$$

likewise, an upper solution $y$ in $C^{1}(J, R)$ to the Non-HFIDE (19) is introduced on J by inverting the above inequalities.

\subsection{Existence theorem}

In the following hypothesis relating to our further discussion:

$\left(A_{1}\right)$ There is a constant $M_{f}>0$ such that $|f(t, u, v)| \leq M_{f}, \forall t \in J$ and $u$ in $R$.

$\left(A_{2}\right)$ The function $f(t, u, v)$ is monotone increasing in $u$ and $v$ for any $t$ in $J$.

$\left(A_{3}\right)$ The function $g(t, u)$ is monotone increasing in $u$ for any $t$ in $J$.

$\left(A_{4}\right)$ The Non HFIDE (1) has a lower solution $z \in C^{1}(J, R)$.

$\left(A_{5}\right)$ There is a constant $L>0$ such that

$$
0 \leq g(t, u)-g(t, v) \leq L(u-v)
$$

for every $t \in J, \quad u, v \in R$, with $u \geq v$.

$\left(A_{6}\right)$ There are D-functions $\Xi_{1}$ and $\Xi_{2}$ such that

$$
0 \leq f\left(t, x_{1}, x_{2}\right)-f\left(t, y_{1}, y_{2}\right) \leq \Xi_{1}\left(x_{1}-y_{1}\right)+\Xi_{2}\left(x_{2}-y_{2}\right)
$$

for all $u_{1}, u_{2}, v_{1}, v_{2} \in R$ with $u_{1} \geq v_{1}$ and $u_{2} \geq v_{2}$. Furthermore

$$
\frac{a^{\alpha}}{\Gamma(\alpha+1)}\left(\Xi_{1}(r)+\Xi_{2}(L a r)\right)<r, \text { for any, } r>0
$$

$\left(A_{7}\right)$ The Non - HFIDE (19) has a lower solution $z \in C^{1}(J, R)$.

The following lemma is important in our next work and its proof is clear by a direct verification (by using the definition Riemann \& Liouville differential operator).

Lemma 3.4 Let $h: J \rightarrow R$, be an integrablea function then $u \in C^{1}(J, R)$ is a solution of NonHFIDE

$$
\begin{aligned}
\left(D^{\alpha} u\right)(t)+\lambda u(t) & =h(t), \quad t \in J \\
u\left(t_{0}\right) & =B_{0} \in R
\end{aligned}
$$

if and only if it is a solution of the folloeing nonlinear integral equation

$$
u(t)=c e^{-\lambda \frac{t^{\alpha}}{\alpha !}}+e^{-\lambda \frac{t^{\alpha}}{\alpha !}} \int_{t_{0}}^{t} \frac{(t-s)^{\alpha-1}}{\Gamma(\alpha)} e^{\lambda \frac{s^{\alpha}}{\alpha !}} h(s) d s, \quad t \in J
$$

where $c=B_{0} e^{\frac{\lambda t_{0}^{\alpha}}{\Gamma(\alpha)}}$ is a constant. 
Theorem 3.5 Let the conditions $\left(A_{1}-A_{4}\right)$ be held. Then the Non-HFIDE (4) has a solution $u^{*}$ on $J$ and the sequence $\left\{u_{n}\right\}_{n=1}^{\infty}$ the successive approximations, defined by

$$
\begin{aligned}
u_{1}(t) & =y(t), \\
u_{n+1}(t) & =c e^{-\lambda \frac{t^{\alpha}}{\alpha !}}+e^{-\lambda \frac{t^{\alpha}}{\alpha !}} \int_{t_{0}}^{t} \frac{(t-s)^{\alpha-1}}{\Gamma(\alpha)} e^{\lambda s^{\alpha} \alpha !} f\left(s, u_{n}(s), \int_{t_{0}}^{s} \frac{(s-\xi)^{\alpha-1}}{\Gamma(\alpha)} g\left(\xi, u_{n}(\xi)\right) d \xi\right) d s
\end{aligned}
$$

for all $t$ in $R$, converges monotonically to $u^{*}$.

Proof. From Lemma 3.4, the Non - HfIDE (4) is equivalent to the nonlinear integral equation

$$
u(t)=c e^{-\lambda \frac{t^{\alpha}}{\alpha !}}+e^{-\lambda \frac{t^{\alpha}}{\alpha !}} \int_{t_{0}}^{t} \frac{(t-s)^{\alpha-1}}{\Gamma(\alpha)} e^{\lambda \frac{s^{\alpha}}{\alpha !}} f\left(s, u(s), \int_{t_{0}}^{s} \frac{(s-\xi)^{\alpha-1}}{\Gamma(\alpha)} g(\xi, u(\xi)) d \xi\right) d s, \quad t \in J .
$$

Set $E=C(J, R)$. Then, by Lemma 3.3 it follows that each compact chain in $E$ has the property of compatibility with respect to the order relation $\preceq$ and the norm $\|\cdot\|$ in $E$. We introduce the operator $T$ by

$$
T u(t)=c e^{-\lambda \frac{t^{\alpha}}{\alpha !}}+e^{-\lambda \frac{t^{\alpha}}{\alpha !}} \int_{t_{0}}^{t} \frac{(t-s)^{\alpha-1}}{\Gamma(\alpha)} e^{\lambda \frac{s^{\alpha}}{\alpha !}} f\left(s, u(s), \int_{t_{0}}^{s} \frac{(s-\xi)^{\alpha-1}}{\Gamma(\alpha)} g(\xi, u(\xi)) d \xi\right) d s, \quad t \in J .
$$

Since $T u$ is continuous, then $T u \in E$. That is, $T$ maps $E$ into itself. The Non - HFIDE (4) is then equivalent to the operator equation

$$
T u(t)=u(t), \quad t \in J
$$

Through a series of steps, we must prove that the operator $T$ fulfills all the conditions of Theorem 2.1.

Step $I$ : Let $T$ be increasing on $E$. Let $u, v$ in $E$ with $u \leq v$. Then, from $\left(A_{2}\right)$, we get

$$
\begin{aligned}
T u(t)= & c e^{-\lambda \frac{t^{\alpha}}{\alpha !}}+e^{-\lambda \frac{t^{\alpha}}{\alpha !}} \int_{t_{0}}^{t} \frac{(t-s)^{\alpha-1}}{\Gamma(\alpha)} e^{\lambda \frac{s^{\alpha}}{\alpha !}} f\left(s, u(s), \int_{t_{0}}^{s} \frac{(s-\xi)^{\alpha-1}}{\Gamma(\alpha)} g(\xi, u(\xi)) d T\right) d s \\
& \leq c e^{-\lambda \frac{t^{\alpha}}{\alpha !}}+e^{-\lambda \frac{t^{\alpha}}{\alpha !}} \int_{t_{0}}^{t} \frac{(t-s)^{\alpha-1}}{\Gamma(\alpha)} e^{\lambda \frac{s^{\alpha}}{\alpha !}} f\left(s, v(s), \int_{t_{0}}^{s} \frac{(s-\xi)^{\alpha-1}}{\Gamma(\alpha)} g(\xi, v(\xi)) d \xi\right) d s \\
& =T v(t)
\end{aligned}
$$

for all $t$ in $J$. This proves that $T$ is a increasing operator on $E$.

Step $I I$ : Let $T$ be partially continuous on $E$. $\left\{u_{n}\right\}$ is a chain points sequences $C$ in $E$ such that $u_{n} \rightarrow u, \forall n \in N$. Then, by the controlled convergence theorem,

$$
\begin{aligned}
\lim _{n \rightarrow \infty} T u_{n}(t)= & \lim _{n \rightarrow \infty}\left(c e^{-\lambda \frac{t^{\alpha}}{\alpha !}}+e^{-\lambda \frac{t^{\alpha}}{\alpha !}} \int_{t_{0}}^{t} \frac{(t-s)^{\alpha-1}}{\Gamma(\alpha)} e^{\lambda \frac{s^{\alpha}}{\alpha !}} f\left(s, u_{n}(s), \int_{t_{0}}^{s} \frac{(s-\xi)^{\alpha-1}}{\Gamma(\alpha)} g\left(\xi, u_{n}(\xi)\right) d \xi\right) d s\right) \\
& =c e^{-\lambda \frac{t^{\alpha}}{\alpha !}}+e^{-\lambda \frac{t^{\alpha}}{\alpha !}} \int_{t_{0}}^{t} \frac{(t-s)^{\alpha-1}}{\Gamma(\alpha)} e^{\lambda \frac{s^{\alpha}}{\alpha !}}\left(\lim _{n \rightarrow \infty} f\left(s, u_{n}(s), \int_{t_{0}}^{s} \frac{(s-\xi)^{\alpha-1}}{\Gamma(\alpha)} g\left(\xi, u_{n}(\xi)\right) d \xi\right) d s\right) \\
& =c e^{-\lambda \frac{t^{\alpha}}{\alpha !}}+e^{-\lambda \frac{t^{\alpha}}{\alpha !}} \int_{t_{0}}^{t} \frac{(t-s)^{\alpha-1}}{\Gamma(\alpha)} e^{\lambda \frac{s^{\alpha}}{\alpha !}} f\left(s, u(s), \int_{t_{0}}^{s} \frac{(s-\xi)^{\alpha-1}}{\Gamma(\alpha)} g(\xi, u(\xi)) d \xi\right) d s \\
& =T u(t)
\end{aligned}
$$

for every $t$ in $J$. This proves that $\left\{T u_{n}\right\}$ converges to $T u$ point-wise on $J$. Therefore, we prove that $\left\{T u_{n}\right\}$ is an equi-continuous sequence of functions in $E$. Let $t_{1}, t_{2}$ in $J$ with $t_{1}<t_{2}$. Then

$$
\begin{gathered}
\left|T u_{n}\left(t_{2}\right)-T u_{n}\left(t_{1}\right)\right| \leq c\left|e^{-\lambda \frac{t_{1}^{\alpha}}{\alpha !}}-e^{-\lambda \frac{t_{2}^{\alpha}}{\alpha !}}\right| \\
++e^{-\lambda \frac{t_{2}^{\alpha}}{\alpha !}} \int_{t_{0}}^{t_{2}} \frac{\left(t_{2}-s\right)^{\alpha-1}}{\Gamma(\alpha)} e^{\lambda \frac{s^{\alpha}}{\alpha !}} f\left(s, u_{n}(s), \int_{t_{0}}^{s} \frac{(s-\xi)^{\alpha-1}}{\Gamma(\alpha)} g\left(\xi, u_{n}(\xi)\right) d \xi\right) d s
\end{gathered}
$$




$$
\begin{aligned}
& -e^{-\lambda \frac{t_{1}^{\alpha}}{\alpha !}} \int_{t_{0}}^{t_{1}} \frac{\left(t_{1}-s\right)^{\alpha-1}}{\Gamma(\alpha)} e^{\lambda \frac{s^{\alpha}}{\alpha !}} f\left(s, u_{n}(s), \int_{t_{0}}^{s} \frac{(s-\xi)^{\alpha-1}}{\Gamma(\alpha)} g\left(\xi, u_{n}(\xi)\right) d \xi\right) d s \mid \\
& \leq c\left|e^{-\lambda \frac{t_{1}^{\alpha}}{\alpha !}}-e^{-\lambda \frac{t_{2}^{\alpha}}{\alpha !}}\right|+\mid e^{-\lambda \frac{t_{2}^{\alpha}}{\alpha !}} \int_{t_{0}}^{t_{2}} \frac{\left(t_{2}-s\right)^{\alpha-1}}{\Gamma(\alpha)} e^{\lambda \frac{s^{\alpha}}{\alpha !}} f\left(s, u_{n}(s), \int_{t_{0}}^{s} \frac{(s-\xi)^{\alpha-1}}{\Gamma(\alpha)} g\left(\xi, u_{n}(\xi)\right) d \xi\right) d s \\
& -e^{-\lambda \frac{t_{1}^{\alpha}}{\alpha !}} \int_{t_{0}}^{t_{2}} \frac{\left(t_{1}-s\right)^{\alpha-1}}{\Gamma(\alpha)} e^{\lambda \frac{s^{\alpha}}{\alpha !}} f\left(s, u_{n}(s), \int_{t_{0}}^{s} \frac{(s-\xi)^{\alpha-1}}{\Gamma(\alpha)} g\left(\xi, u_{n}(\xi)\right) d \xi\right) d s \mid \\
& +\mid e^{-\lambda \frac{t_{\alpha}^{\alpha}}{\alpha !}} \int_{t_{0}}^{t_{2}} \frac{\left(t_{1}-s\right)^{\alpha-1}}{\Gamma(\alpha)} e^{\lambda \frac{s^{\alpha}}{\alpha !}} f\left(s, u_{n}(s), \int_{t_{0}}^{s} \frac{(s-\xi)^{\alpha-1}}{\Gamma(\alpha)} g\left(\xi, u_{n}(\xi)\right) d \xi\right) d s \\
& -e^{-\lambda \frac{t_{1}^{\alpha}}{\alpha !}} \int_{t_{0}}^{t_{1}} \frac{\left(t_{1}-s\right)^{\alpha-1}}{\Gamma(\alpha)} e^{\lambda \frac{s^{\alpha}}{\alpha !}} f\left(s, u_{n}(s), \int_{t_{0}}^{s} \frac{(s-\xi)^{\alpha-1}}{\Gamma(\alpha)} g\left(\xi, u_{n}(\xi)\right) d \xi\right) d s \mid \\
& \leq c\left|e^{-\lambda \frac{t_{1}^{\alpha}}{\alpha !}}-e^{-\lambda \frac{t_{2}^{\alpha}}{\alpha !}}\right| \\
& +\left|e^{-\lambda \frac{t_{2}^{\alpha}}{\alpha !}}-e^{-\lambda \frac{t_{1}^{\alpha}}{\alpha !}} \| \int_{t_{0}}^{t_{2}} \frac{\left(t_{2}-s\right)^{\alpha-1}-\left(t_{1}-s\right)^{\alpha-1}}{\Gamma(\alpha)} e^{\lambda \frac{s^{\alpha}}{\alpha !}} f\left(s, u_{n}(s), \int_{t_{0}}^{s} \frac{(s-\xi)^{\alpha-1}}{\Gamma(\alpha)} g\left(\xi, u_{n}(\xi)\right) d \xi\right) d s\right| \\
& +e^{-\lambda \frac{t_{1}^{\alpha}}{\alpha !}} \mid \int_{t_{0}}^{t_{2}} \frac{\left(t_{1}-s\right)^{\alpha-1}}{\Gamma(\alpha)} e^{\lambda \frac{s^{\alpha}}{\alpha !}} f\left(s, u_{n}(s), \int_{t_{0}}^{s} \frac{(s-\xi)^{\alpha-1}}{\Gamma(\alpha)} g\left(\xi, x_{n}(\xi)\right) d \xi\right) d s \\
& -\int_{t_{0}}^{t_{1}} \frac{\left(t_{1}-s\right)^{\alpha-1}}{\Gamma(\alpha)} e^{\lambda \frac{s^{\alpha}}{\alpha !}} f\left(s, u_{n}(s), \int_{t_{0}}^{s} \frac{(s-\xi)^{\alpha-1}}{\Gamma(\alpha)} g\left(\xi, u_{n}(\xi)\right) d \xi\right) d s \mid \\
& \leq c\left|e^{-\lambda \frac{t_{1}^{\alpha}}{\alpha !}}-e^{-\lambda \frac{t_{2}^{\alpha}}{\alpha !}}\right|+\left|e^{-\lambda \frac{t_{2}^{\alpha}}{\alpha !}}-e^{-\lambda \frac{t_{1}^{\alpha}}{\alpha !}}\right| \\
& \left|\int_{t_{0}}^{t_{0}+a} \frac{\left(t_{2}-s\right)^{\alpha-1}-\left(t_{1}-s\right)^{\alpha-1}}{\Gamma(\alpha)} e^{\lambda s^{\alpha}} \alpha ! f\left(s, u_{n}(s), \int_{t_{0}}^{s} \frac{(s-\xi)^{\alpha-1}}{\Gamma(\alpha)} g\left(\xi, u_{n}(\xi)\right) d \xi\right) d s\right| \\
& \left|\int_{t_{1}}^{t_{2}} \frac{\left(t_{1}-s\right)^{\alpha-1}}{\Gamma(\alpha)} e^{\lambda s^{\alpha}} \alpha\left(s, u_{n}(s), \int_{t_{0}}^{s} \frac{(s-\xi)^{\alpha-1}}{\Gamma(\alpha)} g\left(\xi, u_{n}(\xi)\right) d \xi\right) d s\right| \\
& \leq\left|e^{-\lambda \frac{t_{1}^{\alpha}}{\alpha !}}-e^{-\lambda \frac{t_{2}^{\alpha}}{\alpha !}}\right|\left(c+\frac{M_{f}}{\Gamma(\alpha)} \int_{t_{0}}^{t_{0}+a}\left(t_{2}-s\right)^{(\alpha-1)}-\left(t_{1}-s\right)^{\alpha-1} e^{\lambda \frac{s^{\alpha}}{\alpha !}} d s\right) \\
& +\frac{M_{f}}{\Gamma(\alpha)}\left|\int_{t_{1}}^{t_{2}}-\left(t_{1}-s\right)^{\alpha-1} e^{\lambda \frac{s^{\alpha}}{\alpha !}}\right| d s \\
& \leq\left|e^{-\lambda \frac{t_{1}^{\alpha}}{\alpha !}}-e^{-\lambda \frac{t_{2}^{\alpha}}{\alpha !}}\right|\left(c+\frac{M_{f}}{\Gamma(\alpha)} \int_{t_{0}}^{t_{0}+a}\left(\left(t_{2}-s\right)^{(\alpha-1)}-\left(t_{1}-s\right)^{\alpha-1}\right) e^{\lambda \frac{s^{\alpha}}{\alpha !}} d s\right)+\frac{M_{f}}{\Gamma(\alpha)} \mid\left(p\left(t_{1}\right)-p\left(t_{2}\right) \mid\right. \\
& \leq\left|e^{-\lambda \frac{t_{1}^{\alpha}}{\alpha !}}-e^{-\lambda \frac{t_{2}^{\alpha}}{\alpha !}}\right|\left(c+\frac{M_{f} \cdot e^{\lambda\left(t_{0}+a\right)^{\alpha-1}}}{\Gamma(\alpha)}\right)+\frac{M_{f}}{\Gamma(\alpha)} \mid\left(p\left(t_{1}\right)-p\left(t_{2}\right) \mid\right.
\end{aligned}
$$

where, $p(t)=\int_{t_{0}}^{t} \frac{\left(t_{0}+a-s\right)^{\alpha-1}}{\Gamma(\alpha)} e^{\lambda \frac{s^{\alpha}}{\alpha !}} d s, \quad t \in J$.

Step III: Let $T$ be a operator of partially compact on $E$ and $C$ is an arbitrary chain in $E$. Then we prove that $T(C)$ is a equi-continuous an uniformly bounded set in $E$. Firstly, we prove that $T(C)$ is uniformly bounded. We put $u \in C$ is arbitrary. Then,

$$
\begin{gathered}
|T u(t)| \leq\left|c e^{-\lambda \frac{t^{\alpha}}{\alpha !}}\right|+\left|e^{-\lambda \frac{t^{\alpha}}{\alpha !}} \int_{t_{0}}^{t} \frac{(t-s)^{\alpha-1}}{\Gamma(\alpha)} e^{\lambda \frac{s^{\alpha}}{\alpha !}} f\left(s, u(s), \int_{t_{0}}^{s} \frac{(s-\xi)^{\alpha-1}}{\Gamma(\alpha)} g(\xi, u(\xi)) d \xi\right) d s\right| \\
\leq|c|+\frac{a^{\alpha} M_{f}}{\Gamma(\alpha+1)}=r
\end{gathered}
$$


for every $t$ in $J$. We are taking the supremum over $t$, we get $\|T u\| \leq r$ for every $u \in C$. Thus $T(C) \prec E$ is a uniformly bounded. Therefore, we will prove that $T(C)$ is an equicontinuous set in $E$. We put $t_{1}, t_{2} \in J$ is arbitrary with $t_{1}<t_{2}$. Then

$$
\begin{aligned}
& \left|T u\left(t_{2}\right)-T u\left(t_{1}\right)\right| \leq c\left|e^{-\lambda \frac{t_{1}^{\alpha}}{\alpha !}}-e^{-\lambda \frac{t_{2}^{\alpha}}{\alpha !}}\right| \\
& +\mid e^{-\lambda \frac{t_{2}^{\alpha}}{\alpha !}} \int_{t_{0}}^{t_{2}} \frac{\left(t_{2}-s\right)^{\alpha-1}}{\Gamma(\alpha)} e^{\lambda s} f\left(s, u(s), \int_{t_{0}}^{s} \frac{(s-\xi)^{\alpha-1}}{\Gamma(\alpha)} g(\xi, u(\xi)) d \xi\right) d s \\
& -e^{-\lambda \frac{t_{1}^{\alpha}}{\alpha !}} \int_{t_{0}}^{t_{1}} \frac{\left(t_{1}-s\right)^{\alpha-1}}{\Gamma(\alpha)} e^{\lambda \frac{s^{\alpha}}{\alpha !}} f\left(s, u(s), \int_{t_{0}}^{s} \frac{(s-\xi)^{\alpha-1}}{\Gamma(\alpha)} g(\xi, u(\xi)) d \xi\right) d s \mid \\
& \leq c\left|e^{-\lambda \frac{t_{1}^{\alpha}}{\alpha !}}-e^{-\lambda \frac{t_{2}^{\alpha}}{\alpha !}}\right|+\mid e^{-\lambda \frac{t_{2}^{\alpha}}{\alpha !}} \int_{t_{0}}^{t_{2}} \frac{\left(t_{2}-s\right)^{\alpha-1}}{\Gamma(\alpha)} e^{\lambda \frac{s^{\alpha}}{\alpha !}} f\left(s, u(s), \int_{t_{0}}^{s} \frac{(s-\xi)^{\alpha-1}}{\Gamma(\alpha)} g(\xi, u(\xi)) d \xi\right) d s \\
& -e^{-\lambda \frac{t_{1}^{\alpha}}{\alpha !}} \int_{t_{0}}^{t_{2}} \frac{\left(t_{1}-s\right)^{\alpha-1}}{\Gamma(\alpha)} e^{\lambda \frac{s^{\alpha}}{\alpha !}} f\left(s, u(s), \int_{t_{0}}^{s} \frac{(s-\xi)^{\alpha-1}}{\Gamma(\alpha)} g(\xi, u(\xi)) d \xi\right) d s \mid \\
& +\mid e^{-\lambda \frac{t_{1}^{\alpha}}{\alpha !}} \int_{t_{0}}^{t_{2}} \frac{\left(t_{1}-s\right)^{\alpha-1}}{\Gamma(\alpha)} e^{\lambda \frac{s^{\alpha}}{\alpha !}} f\left(s, u(s), \int_{t_{0}}^{s} \frac{(s-\xi)^{\alpha-1}}{\Gamma(\alpha)} g(\xi, u(\xi)) d \xi\right) d s \\
& -e^{-\lambda \frac{t_{1}^{\alpha}}{\alpha !}} \int_{t_{0}}^{t_{1}} \frac{\left(t_{1}-s\right)^{\alpha-1}}{\Gamma(\alpha)} e^{\lambda \frac{s^{\alpha}}{\alpha !}} f\left(s, u(s), \int_{t_{0}}^{s} \frac{(s-\xi)^{\alpha-1}}{\Gamma(\alpha)} g(\xi, u(\xi)) d \xi\right) d s \mid \\
& \leq c\left|e^{-\lambda \frac{t_{1}^{\alpha}}{\alpha !}}-e^{-\lambda \frac{t_{2}^{\alpha}}{\alpha !}}\right| \\
& +\left|e^{-\lambda \frac{t_{2}^{\alpha}}{\alpha !}}-e^{-\lambda \frac{t_{1}^{\alpha}}{\alpha !}} \| \int_{t_{0}}^{t_{2}} \frac{\left(t_{2}-s\right)^{\alpha-1}-\left(t_{1}-s\right)^{\alpha-1}}{\Gamma(\alpha)} e^{\lambda \frac{s^{\alpha}}{\alpha !}} f\left(s, u(s), \int_{t_{0}}^{s} \frac{(s-\xi)^{\alpha-1}}{\Gamma(\alpha)} g(\xi, u(\xi)) d \xi\right) d s\right| \\
& +e^{-\lambda \frac{t_{1}^{\alpha}}{\alpha !}} \mid \int_{t_{0}}^{t_{2}} \frac{\left(t_{1}-s\right)^{\alpha-1}}{\Gamma(\alpha)} e^{\lambda \frac{s^{\alpha}}{\alpha !}} f\left(s, u(s), \int_{t_{0}}^{s} \frac{(s-\xi)^{\alpha-1}}{\Gamma(\alpha)} g(\xi, u(\xi)) d \xi\right) d s \\
& -\int_{t_{0}}^{t_{1}} \frac{\left(t_{1}-s\right)^{\alpha-1}}{\Gamma(\alpha)} e^{\lambda \frac{s^{\alpha}}{\alpha !}} f\left(s, u(s), \int_{t_{0}}^{s} \frac{(s-\xi)^{\alpha-1}}{\Gamma(\alpha)} g(\xi, u(\xi)) d \xi\right) d s \mid \\
& \leq c\left|e^{-\lambda \frac{t_{1}^{\alpha}}{\alpha !}}-e^{-\lambda \frac{t_{2}^{\alpha}}{\alpha !}}\right|+\left|e^{-\lambda \frac{t_{2}^{\alpha}}{\alpha !}}-e^{-\lambda \frac{t_{1}^{\alpha}}{\alpha !}}\right| \\
& \left|\int_{t_{0}}^{t_{0}+a} \frac{\left(t_{2}-s\right)^{\alpha-1}-\left(t_{1}-s\right)^{\alpha-1}}{\Gamma(\alpha)} e^{\lambda \frac{s^{\alpha}}{\alpha !}} f\left(s, u(s), \int_{t_{0}}^{s} \frac{(s-\xi)^{\alpha-1}}{\Gamma(\alpha)} g(\xi, u(\xi)) d \xi\right) d s\right| \\
& \left|\int_{t_{1}}^{t_{2}} \frac{\left(t_{1}-s\right)^{\alpha-1}}{\Gamma(\alpha)} e^{\lambda \frac{s^{\alpha}}{\alpha !}} f\left(s, u(s), \int_{t_{0}}^{s} \frac{(s-\xi)^{\alpha-1}}{\Gamma(\alpha)} g(\xi, u(\xi)) d \xi\right) d s\right| \\
& \leq\left|e^{-\lambda \frac{t_{1}^{\alpha}}{\alpha !}}-e^{-\lambda \frac{t_{2}^{\alpha}}{\alpha !}}\right|\left(c+\frac{M_{f}}{\Gamma(\alpha)} \int_{t_{0}}^{t_{0}+a}\left(t_{2}-s\right)^{(\alpha-1)}-\left(t_{1}-s\right)^{\alpha-1} e^{\lambda \frac{s^{\alpha}}{\alpha !}} d s\right) \\
& +\frac{M_{f}}{\Gamma(\alpha)} \int_{t_{1}}^{t_{2}}\left(t_{1}-s\right)^{\alpha-1} e^{\lambda \frac{s^{\alpha}}{\alpha !}} d s \\
& \leq\left|e^{-\lambda \frac{t_{1}^{\alpha}}{\alpha !}}-e^{-\lambda \frac{t_{2}^{\alpha}}{\alpha !}}\right|\left(c+\frac{M_{f}}{\Gamma(\alpha)} \int_{t_{0}}^{t_{0}+a}\left(c\left(t_{2}-s\right)^{(\alpha-1)}-\left(t_{1}-s\right)^{\alpha-1}\right) e^{\lambda \frac{s^{\alpha}}{\alpha !}} d s\right)+\frac{M_{f}}{\Gamma(\alpha)} \mid\left(p\left(t_{1}\right)-p\left(t_{2}\right) \mid\right.
\end{aligned}
$$

where, $p(t)=\int_{t_{0}}^{t}\left(t_{0}+a-s\right)^{\alpha-1} e^{\lambda \frac{s^{\alpha}}{\alpha !}} d s, \quad t \in J$.

Since the functions $t \rightarrow(t-s)^{\alpha-1}$ and $t \rightarrow p(t)$ are uniformly continuous on compact $J=\left[t_{0}, t_{0}+a\right]$, we have that $\left|T u\left(t_{2}\right)-T u\left(t_{1}\right)\right| \rightarrow 0$ as $t_{2} \rightarrow t_{1}$, uniformly for every $u \in C$. This proves that $T(C)$ is an equi-continuous set in $E$. Thus $T(C) \prec E$ is compact and consequently $T$ is a operator of partially compact on $E \rightarrow E$. 
Step $I V: z$ fulfills the operator inequality $z \leq T z$.

from the supposition $\left(A_{4}\right)$ Achieves, $y$ is a lower solution of Non-HFIDE (4) introduced on $J$. Thus

$$
\left(D^{\alpha} z\right)(t)+\lambda z(t) \leq f\left(t, z(t), \int_{t_{0}}^{t} \frac{(t-s)^{\alpha-1}}{\Gamma(\alpha)} g(s, z(s)) d s\right),
$$

and

$$
z\left(t_{0}\right) \leq B_{0} \in R
$$

for every $t$ in $J$. The integrating (12) from $t_{0}$ to $t$, we have

$$
z(t) \leq c e^{-\lambda \frac{t^{\alpha}}{\alpha !}}+e^{-\lambda \frac{t^{\alpha}}{\alpha !}} \int_{t_{0}}^{t} \frac{(t-s)^{\alpha-1}}{\Gamma(\alpha)} e^{\lambda \frac{s^{\alpha}}{\alpha !}} f\left(s, z(s), \int_{t_{0}}^{s} \frac{(s-\xi)^{\alpha-1}}{\Gamma(\alpha)} g(\xi, z(\xi)) d \xi\right) d s
$$

for every $t$ in $J$. This prove that $z$ is a lower solution of the operator equation $u=T u$.

Thus $T$ satisfies all the conditions of Theorem 2.1, and Remark 2.1 and we can deduce which the operator equation $T u=u$ contain of a solution. Therefore, the integral equation and Non $-H f I D E$ (4) have a solution $u^{*}$ introduced in $J$. Moreover, the sequence $\left\{u_{n}\right\}$ of successive approximations introduced by Eq. (8) converges monotonically to $u^{*}$. Thus we have completed the proof.

Remark 3.2 The inference of theorem 3.5 also still true if we substitute condition $\left(A_{4}\right)$ with $\left(B_{4}\right)$ The Non - H IIDE (4) contain a upper solution $v \in C^{1}(J, R)$.

Example 3.1 The interval $J=[0,1]$ is a closed and bounded, we consider the Non-HFIDE

$$
\begin{gathered}
\left(D^{\frac{3}{2}} u\right)(t)+u(t)=\tan h u(t)+\tan h \int_{0}^{t} \frac{(t-s)^{\frac{1}{2}}}{\Gamma\left(\frac{3}{2}\right)} \sin h u(s) d s, \\
u(0)=1 .
\end{gathered}
$$

We applied the theorem 3.5, we get $\lambda=1, c=1, g(t, u)=\sinh u$ and $f(t, u, v)=\tanh u+\tanh v$. Obviously, the functions $f$ and $g$ are continuous on $J \times R$, and $f$ achieves $\left(A_{1}\right)$ with $M_{f}=2$. Furthermore, $g(t, u)$ is increasing in $u$ for any $t$ in $J$, and $f(t, u, v)$ is increasing in $u$ and $v$ for any $t$ in $J$, thus conditions $\left(A_{2}\right)$ and $\left(A_{3}\right)$ are achieved. Finally, the Non-HFIDE(15) has a lower solution $z$ defined by

$$
z(t)=\frac{2}{\Gamma\left(\frac{3}{2}\right)}\left(e^{\frac{-t^{\frac{1}{2}}}{\Gamma\left(\frac{3}{2}\right)}}-1\right)
$$

on $J$. Hence, all the assumption of Theorem 2.1 are achieved, and thus Non-HFIDE(15) has a solution $u^{*}$ introduced on $J$, and the sequence $\left\{u_{n}\right\}$, introduced by

$$
\begin{gathered}
u_{1}(t)=y(t), \\
u_{n+1}(t)=e^{-\frac{t^{\alpha}}{\alpha !}}+e^{-\frac{t^{\alpha}}{\alpha !}} \int_{0}^{t} \frac{(t-s)^{\frac{1}{2}}}{\Gamma\left(^{\frac{3}{2}}\right)} e^{\frac{s^{\alpha}}{\alpha !}} \tan h u_{n}(s) d s \\
+e^{-\frac{t^{\alpha}}{\alpha !}} \int_{0}^{t} \frac{(t-s)^{\frac{1}{2}}}{\Gamma\left(\frac{3}{2}\right)} e^{\frac{s^{\alpha}}{\alpha !}} \tan h\left(\int_{0}^{s} \frac{(s-\xi)^{\frac{1}{2}}}{\Gamma\left(\frac{3}{2}\right)} \sinh u_{n}(\xi) d \xi\right) d s
\end{gathered}
$$

for all $t \in J$ and where $\Gamma\left(\frac{3}{2}\right)=\frac{3 \sqrt{\pi}}{2}$, converges monotonically to $u^{*}$. 


\subsection{Uniqueness theorem}

In this section, we investigate a uniqueness theorem for the Non-HfIDE (4) by using the weaker partially Lipschitz condition.

Theorem 3.6 A suppose that conditions $\left(A_{4}\right)-\left(A_{6}\right)$ achieve. Then the Non-HfIDE (4) contain of an unique solution $u^{*}$ introduced on $J$, and the sequence $\left\{u_{n}\right\}$ of successive approximations introduced by the Eq. (8) converges monotonically to $u^{*}$.

Proof. Set $E=C(J, R)$. Obviously, $E$ is a lattice with respect to the order relation $\preceq$ and thus upper and lower bounds there is for all pair of elements in $E$. We introduce the operator $T$ by (10). Then, the Non-HfIDE (4) is equivalent to the operator equation (11). We must prove that $T$ fulfills all the conditions of Theorem 2.1.

Obviously, $T$ is a increasing operator from $E \rightarrow E$. We want to prove that the operator $T$ is a partially nonlinear D-contraction on $E$, thus let $u, v \in E$ with $u \geq v$. Then, by $\left(A_{5}\right)$ and $\left(A_{6}\right)$

$$
\begin{aligned}
& |T x(t)-T v(t)| \leq \mid e^{-\lambda \frac{t^{\alpha}}{\alpha !}} \int_{t_{0}}^{t} \frac{(t-s)^{\alpha-1}}{\Gamma(\alpha)} e^{\lambda \frac{s^{\alpha}}{\alpha !}} f\left(s, u(s), \int_{t_{0}}^{s} \frac{(s-\xi)^{\alpha-1}}{\Gamma(\alpha)} g(\xi, u(\xi)) d \xi\right) d s \\
& -e^{-\lambda \frac{t^{\alpha}}{\alpha !}} \int_{t_{0}}^{t} \frac{(t-s)^{\alpha-1}}{\Gamma(\alpha)} e^{\lambda \frac{s^{\alpha}}{\alpha !}} f\left(s, v(s), \int_{t_{0}}^{s} \frac{(s-\xi)^{\alpha-1}}{\Gamma(\alpha)} g(\xi, v(\xi)) d \xi\right) d s \mid \\
& \leq e^{-\lambda \frac{t^{\alpha}}{\alpha !}} \int_{t_{0}}^{t} \frac{(t-s)^{\alpha-1}}{\Gamma(\alpha)} e^{\lambda \frac{s^{\alpha}}{\alpha !}} \mid f\left(s, u(s), \int_{t_{0}}^{s} \frac{(s-\xi)^{\alpha-1}}{\Gamma(\alpha)} g(\xi, u(\xi)) d \xi\right) \\
& -f\left(s, v(s), \int_{t_{0}}^{s} \frac{(s-\xi)^{\alpha-1}}{\Gamma(\alpha)} g(\xi, v(\xi)) d \xi\right) \mid d s \\
& \leq e^{-\lambda \frac{t^{\alpha}}{\alpha !}} \int_{t_{0}}^{t} \frac{(t-s)^{\alpha-1}}{\Gamma(\alpha)} e^{\lambda \frac{s^{\alpha}}{\alpha !}}\left(\xi_{1}(u(s)-v(t))+\xi_{2}\left(c \int_{t_{0}}^{s} \frac{(s-\xi)^{\alpha-1}}{\Gamma(\alpha)} g(\xi, u(\xi)) d \xi-g(\xi, v(\xi))\right) d \xi\right) d s \\
& \leq e^{-\lambda \frac{t^{\alpha}}{\alpha !}} \int_{t_{0}}^{t} \frac{(t-s)^{\alpha-1}}{\Gamma(\alpha)} e^{\frac{-\lambda}{\alpha !}\left(t^{\alpha}-s^{\alpha}\right)}\left(\xi_{1}(u(s)-v(s))+\xi_{2} \int_{t_{0}}^{s} \frac{(s-\Xi)^{\alpha-1}}{\Gamma(\alpha)} L(u(\xi)-v(\xi)) d \xi\right) d s \\
& \leq \frac{1}{\Gamma(\alpha)} \int_{t_{0}}^{t}(t-s)^{\alpha-1} e^{\frac{-\lambda}{\alpha !}}\left(t^{\alpha}-s^{\alpha}\right)\left(\xi_{1}(\|u-v\|)+\xi_{2} \frac{b^{\alpha}}{\Gamma(\alpha+1)} L a\|u-v\|\right) d s \\
& \leq \xi\|u-v\|
\end{aligned}
$$

for every $t \in J$, where $\xi(r)=\frac{a^{\alpha}}{\Gamma(\alpha+1)}\left(\xi_{1}(r)+\xi_{2}(L a r)\right)<r, \quad r>0$.

Taking the supremum over $t$, we get

$$
\|T u-T v\| \leq \xi(\|u-v\|)
$$

for every $u, v \in E$, with $u \geq v$. Consequently, $T$ is a partially nonlinear D-contraction in $E$. In addition, as in the proof of Theorem 3.5, we can show that the function $z$ given in condition $\left(A_{4}\right)$ achieves the inequality of the operator $z \leq T z$ in $J$. Now, we apply direction of Theorem 2.1 gives that the Non-HfIDE (4) has a unique solution $u^{*}$, and the sequence $\left\{u_{n}\right\}$ of successive approximations introduced by Eq.(10) converges monotonically to $u^{*}$.

Remark 3.3 The inference of theorem 3.6, also still true if we substitute condition $\left(A_{4}\right)$ with $\left(B_{4}\right)$.

Example 3.2 The interval $J=[0,1]$ is a closed and bounded, we consider the HFIDE

$$
\left(D^{\frac{3}{2}} u\right)(t)+x(t)=\frac{1}{2}\left(\tan ^{-1} u(t)+\tan ^{-1} \int_{t_{0}}^{t} \frac{(t-s)^{\frac{1}{2}}}{\Gamma\left(\frac{3}{2}\right)} g(s, u(s)) d s\right)
$$




$$
u(0)=1
$$

where $g: J \times R \rightarrow R$ is the function introduced by

$$
g(t, u)=\left\{\begin{array}{cr}
1 & \text { if } u \leq 0 \\
1+\frac{u}{1+u} & \text { if } u>0
\end{array}\right.
$$

Hence, $\lambda=1, c=1, f(t, u, v)=\frac{1}{2}\left(\tan ^{-1} u+\tan ^{-1} v\right)$. Obviously, the functions $f$ and $g$ are continuous on $J \times R \times R$ and $J \times R$, consecutively. The function $f$ achieves $\left(A_{1}\right)$ with $M_{f}=\frac{\pi}{2}$ and it is easy to prove that $g$ achieves $\left(A_{5}\right)$ with $L=1$. Further, $f(t, u, v)$ is increasing in $u$ and $v$ for any $t$ in $J$. To prove that $f$ fulfills $\left(A_{6}\right)$ on $J \times R \times R$, let $u_{1}, u_{2}, v_{1}, v_{2} \in R$ be such that $u_{1} \geq v_{1}$ and $u_{2} \geq v_{2}$. Then,

$$
\begin{aligned}
0 \leq & f\left(t, u_{1}, u_{2}\right)-f\left(t, v_{1}, v_{2}\right) \leq \frac{1}{2}\left(\tan ^{-1} u_{1}-\tan ^{-1} v_{1}+\tan ^{-1} u_{2}-\tan ^{-1} v_{2}\right) \\
& \leq \frac{1}{2} \frac{u_{1}-v_{1}}{1+\eta_{1}^{2}}+\frac{1}{2} \frac{u_{2}-v_{2}}{1+\eta_{2}^{2}} \\
& \leq \Xi_{1}\left(u_{1}-v_{1}\right)+\Xi_{2}\left(u_{2}-v_{2}\right)
\end{aligned}
$$

for all $t \in J$ and for some $u_{1}>\eta_{1}>v_{1}$ and $u_{2}>\eta_{2}>v_{2}$, where $\Xi_{1}$ and $\Xi_{2}$ are D-functions introduced by

$$
\Xi_{1}(r)=\frac{r}{1+\eta_{1}^{2}}, \quad \text { and } \quad \Xi_{2}(r)=\frac{r}{1+\eta_{2}^{2}}
$$

for $0<\eta_{1}, \quad \eta_{2}<r$. Furthermore,

$$
\frac{1}{2} \frac{a^{\alpha}}{\Gamma(\alpha+1)}\left(\Xi_{1}(r)+\Xi_{2}(a r)\right)=\frac{r}{1+\eta^{2}}<r
$$

where $\eta=\min \left\{\eta_{1}, \eta_{2}\right\}$.

In the end, the Non-HFIDE (4) has a lower solution $z(t)=\frac{2}{\Gamma\left(\frac{3}{2}\right)}\left(e^{\frac{-t^{\frac{1}{2}}}{\Gamma\left(\frac{3}{2}\right)}}-1\right)$ introduced on $J$. Thus, all the supposition of Theorem 3.6 are fulfilled and thus we conclude that the Non - HFIDE (17) has a unique solution $u^{*}$ introduced on $J$. Moreover, the sequence $\left\{u_{n}\right\}$, introduced by

$$
\begin{gathered}
u_{0}(t)=z(t) \\
x_{n+1}(t)=e^{-\frac{t^{\alpha}}{\alpha !}}+\frac{1}{2} e^{-\frac{t^{\alpha}}{\alpha !}} \int_{0}^{t} \frac{(t-s)^{\alpha-1}}{\Gamma(\alpha)} e^{\frac{s^{\alpha}}{\alpha !}} \tan ^{-1} u_{n}(s) d s \\
+\frac{1}{2} e^{-\frac{t^{\alpha}}{\alpha !}} \int_{0}^{t} \frac{(t-s)^{\alpha-1}}{\Gamma(\alpha)} e^{\frac{s^{\alpha}}{\alpha !} \tan ^{-1}}\left(\int_{0}^{s} \frac{(s-\xi)^{\alpha-1}}{\Gamma(\alpha)} g\left(\xi, u_{n}(\xi)\right) d \xi\right) d s
\end{gathered}
$$

for every $t$ in $J$, converges monotonically to $u^{*}$.

\subsection{The first type linear perturbations}

At times, it is conceivable that the non-linearity of those involved in Non - HFIDE (1) does not fulfill either the supposition of theorem 3.5 or the supposition of theorem 3.6. In spite of, from incising the functions $f_{1}$ and $f_{2}$ of $f$ in the form $f=f_{1}+f_{2}$ fulfill the conditions of Theorems (3.5 and 3.6). Consecutively. in Dhage's terminology [1], the resulting equation is said to be the hybrid integrodifferential equation with the first type linear disturbance. The objective of this section is to get an 
existence result for such an equation.

Given above notations in the sections, we consider the nonlinear hybrid Non-HFIDE

$$
\begin{aligned}
D^{\alpha} u(t)+\lambda u(t)= & f_{1}\left(t, u(t), \int_{t_{0}}^{t} \frac{(t-s)^{\alpha-1}}{\Gamma(\alpha)} g(s, u(s))\right) d s \\
& +f_{2}\left(t, u(t), \int_{t_{0}}^{t} \frac{(t-s)^{\alpha-1}}{\Gamma(\alpha)} g(s, u(s)) d s\right) \\
& u\left(t_{0}\right)=B_{0},
\end{aligned}
$$

for every $t$ in $J$, where $f_{1}, f_{2}: J \times R \times R \rightarrow R$ and $g: J \times R \rightarrow R$ are continuous functions.

From a solution of the Non - HFIDE (19), we denote a function $u \in C^{1}(J, R)$ that fulfills equation (19), where $C^{1}(J, R)$ is the habitual space of continuously differentiable real-valued functions introduced on on $J$.

The Non - HFIDE (19) is called the hybrid fractional integrodifferential equation with a the first type linear perturbation $[12,16]$.

The Non-HFIDE (19) is well recognized in the literature and discussed of the existence and other properties. herein, we prove that existence of solutions by using partially compactness type conditions and the mixed partially Lipschitz.

Theorem 3.7 Assume that $\left(A_{1}\right)-\left(A_{3}\right)$ fulfilled with $f$ and replaced by $f_{2}$, and further let $\left(A_{1}\right),\left(A_{5}\right)$, and $\left(A_{6}\right)$ fulfilled with $f$ and replaced by $f_{1}$. If $\left(A_{7}\right)$ satisfied, then the Non-HFIDE (19) has a solution $u^{*}$ introduced on $J$ and the sequence $\left\{u_{n}\right\}$ of successive approximations, introduced by

$$
\begin{aligned}
u_{1}(t)= & z(t), \\
u_{n+1}(t)= & c e^{-\lambda \frac{t^{\alpha}}{\alpha !}}+e^{-\lambda \frac{t^{\alpha}}{\alpha !}} \int_{t_{0}}^{t} \frac{(t-s)^{\alpha-1}}{\Gamma(\alpha)} e^{\lambda \frac{s^{\alpha}}{\alpha !}} f_{1}\left(s, u_{n}(s), \int_{t_{0}}^{s} \frac{(s-\xi)^{\alpha-1}}{\Gamma(\alpha)} g\left(\xi, u_{n}(\xi)\right) d \xi\right) d s \\
& +e^{-\lambda \frac{t^{\alpha}}{\alpha !}} \int_{t_{0}}^{t} \frac{(t-s)^{\alpha-1}}{\Gamma(\alpha)} e^{\lambda \frac{s^{\alpha}}{\alpha !}} f_{2}\left(s, u_{n}(s), \int_{t_{0}}^{s} \frac{(s-\xi)^{\alpha-1}}{\Gamma(\alpha)} g\left(\xi, u_{n}(\xi)\right) d \xi\right) d s,
\end{aligned}
$$

for $t \in J$, converges monotonically to $u^{*}$.

Proof. Set $E=C(J, R)$. Then, from Lemma 3.4 it follows that each compact chain $C$ in $E$ has the property of compatibility with respect to the order relation $\preceq$ and the norm $\|$.$\| in E$.

According to Lemma 3.4, the Non-HFIDE (19) is equivalent to the nonlinear integral equation

$$
\begin{aligned}
u(t)= & c e^{-\lambda \frac{t^{\alpha}}{\alpha !}}+e^{-\lambda \frac{t^{\alpha}}{\alpha !}} \int_{t_{0}}^{t} \frac{(t-s)^{\alpha-1}}{\Gamma(\alpha)} e^{\lambda \frac{s^{\alpha}}{\alpha !}} f_{1}\left(s, u(s), \int_{t_{0}}^{s} \frac{(s-\xi)^{\alpha-1}}{\Gamma(\alpha)} g(\xi, u(\xi)) d \xi\right) d s, \\
& +e^{-\lambda \frac{t^{\alpha}}{\alpha !}} \int_{t_{0}}^{t} \frac{(t-s)^{\alpha-1}}{\Gamma(\alpha)} e^{\lambda \frac{s^{\alpha}}{\alpha !}} f_{2}\left(s, u(s), \int_{t_{0}}^{s} \frac{(s-\xi)^{\alpha-1}}{\Gamma(\alpha)} g(\xi, u(\xi)) d \xi\right) d s, \quad t \in J .
\end{aligned}
$$

Set $E=C(J, R)$ and introduce the operators $A$ and $B$ on $E$ by

$$
A u(t)=e^{-\lambda \frac{t^{\alpha}}{\alpha !}} \int_{t_{0}}^{t} \frac{(t-s)^{\alpha-1}}{\Gamma(\alpha)} e^{\lambda \frac{s^{\alpha}}{\alpha !}} f_{1}\left(s, u(s), \int_{t_{0}}^{s} \frac{(s-\xi)^{\alpha-1}}{\Gamma(\alpha)} g(\xi, u(\xi)) d \xi\right) d s, \quad t \in J
$$

and

$$
B u(t)=c e^{-\lambda \frac{t^{\alpha}}{\alpha !}}+e^{-\lambda \frac{t^{\alpha}}{\alpha !}} \int_{t_{0}}^{t} \frac{(t-s)^{\alpha-1}}{\Gamma(\alpha)} e^{\lambda \frac{s^{\alpha}}{\alpha !}} f_{2}\left(s, u(s), \int_{t_{0}}^{s} \frac{(s-\xi)^{\alpha-1}}{\Gamma(\alpha)} g(\xi, u(\xi)) d \xi\right) d s, \quad t \in J
$$

Obviously, $A, B: E \rightarrow E$. In addition, Non-HFIDE (19) is equivalent to the operator equation

$$
A u(t)+B u(t)=u(t), \quad t \in J
$$


Following similar arguments to those used in the proofs of Theorems 3.5 and 3.6, we can show that operator $A$ is a nonlinear Dcontraction and partially bounded and $B$ is a partially compact operator and partially continuous in $E$. From the direct application of Theorem 2.2 gives that the operator equation $u \leq A u+B u$ has a solution $u^{*}$. Thus, Non-HFIDE (19) has a sequence $\left\{u_{n}\right\}_{n=1}^{\infty}$, and the solution $u^{*}$ introduced by (20) monotonic converges to $u^{*}$. This completes the proof.

The inference of Theorem 3.7 residues true if we substitute $\left(A_{7}\right)$ with $\left(B_{7}\right)$. The Non $-H F I D E(19)$ has a upper solution $y \in C^{1}(J, R)$.

Example 3.3 The interval $J=[0,1]$ is closed and bounded, we consider the Non - HFIDE,

$$
\begin{gathered}
D^{\frac{1}{2}} u(t)+u(t)=\tan ^{-1} u(t)+\tan h\left(\int_{0}^{t} \frac{(t-s)^{\frac{-1}{2}}}{\Gamma\left(\frac{1}{2}\right)} \sin h u(s) d s\right), \\
u(0)=1 .
\end{gathered}
$$

We applied the Theorem 3.7, we get $\lambda=1, c=1, f_{1}(t, u, v)=\tan ^{-1} u, f_{2}(t, u, v)=\tanh v$ and $g(t, v)=\sinh v$. Therefore the function $f_{1}$ fulfills $\left(A_{1}\right)$ with $M_{f_{1}}=\frac{\pi}{2}$ and also fulfills $\left(A_{6}\right)$ with $\Xi_{1}(r)=\frac{r}{1+\xi^{2}}, 0<\xi<r$ and $\Xi_{2}(r)=0$. Now $f_{2}$ fulfills $\left(A_{1}\right)$ with $M_{f_{2}}=1$ and it is increasing in $v$, thus $\left(A_{2}\right)$ fulfills. Likewise, $g$ fulfills $\left(A_{3}\right)$. In the end, $z(t)=3\left(e^{-t}-1\right)$, for every $t \in J$, is a lower solution of the Non-HFIDE (25) on $J$, and thus $\left(A_{7}\right)$ is fulfilled. Next, by Theorem 3.7, the Non-HFIDE (25) possesses a solution $u^{*}$ on $J$, and the sequence $\left\{u_{n}\right\}_{n=1}^{\infty}$, introduced by

$$
\begin{aligned}
u_{1}(t)= & 3\left(e^{-t}-1\right) \\
u_{n+1}(t)= & e^{-\frac{t^{\alpha}}{\alpha !}}+e^{-\frac{t^{\alpha}}{\alpha !}} \int_{t_{0}}^{t} \frac{(t-s)^{\alpha-1}}{\Gamma(\alpha)} e^{\frac{s^{\alpha}}{\alpha !}} \tan ^{-1} u_{n}(s) d s \\
& +e^{-\frac{t^{\alpha}}{\alpha !}} \int_{0}^{t} \frac{(t-s)^{\alpha-1}}{\Gamma(\alpha)} e^{\frac{s^{\alpha}}{\alpha !}} \tanh \left(\int_{0}^{s} \sinh u_{n}(\xi) d \xi\right) d s,
\end{aligned}
$$

for $t \in J$, converges monotonically to $x^{*}$.

Remark 3.4 We observe that if the Non-HFIDE ((1) or (19)) have a upper y solution, in addition to a lower solution $z$ such that $y \geq z$, then the congruous solutions $u_{*}$ and $u^{*}$ of the Non-HFIDE((1) or(19)) fulfills $u_{*} \leq u^{*}$, and these are the maximum and minimum solutions in the vector segment [z,y] of the Banach space $E=C(J, R)$. In fact, the order relation $\preceq$ introduced by Eq. (5) is equivalent to the order relation introduced by the order etcher

$$
K=\{u \in c(J, R) \mid u(t) \geq 0, \quad \text { for every } t \in J\}
$$

that is a closed set in $C(J, R)$.

\section{References}

[1] Dhage, Bapurao C. and Dhage, Shyam B. and Graef, John R.(2016). Dhage iteration method for initial value problems for nonlinear first order hybrid integrodifferential equations, Journal of Fixed Point Theory and Applications, 18(2) 309-326.

[2] Guo, Boling and Pu, Xueke and Huang, Fenghui (2015). Fractional partial differential equations and their numerical solutions, World Scientific.

[3] Diethelm, Kai(2010). The analysis of fractional differential equations: An application-oriented exposition using differential operators of Caputo type. Springer Science and Business Media.

[4] Kılıçman, Adem and Damag, F.H.M (2018).Some Solution of the Fractional Iterative Integro-Differential Equations. Malaysia Journal of Mathematical Sciences, 12(1).121-141. 
[5] Kreyszig, Erwin (1978). Introductory functional analysis with applications. wiley New York. 1.

[6] Damag, F., Kılıçman, A., Dutta, H., and Ibrahim, R. (2019). A Note on the Lower and Upper Solutions of Hybrid-Type Iterative Fractional Differential Equations. National Academy Science Letters. $10.1007 / \mathrm{s} 40009-019-00863-5$.

[7] Zeidler, Eberhard (2013).Nonlinear Functional Analysis and Its Applications: II/B: Nonlinear Monotone Operators. Springer Science \& Business Media.

[8] Damag, Faten Hasan Mohammed(2017). Existences and uniqueness of solutions for some Classesof Iterative Fractional Functional Integral Differential Equations, psasir.upm.edu.my.

[9] Lakshmikantham, V and Vatsala, AS (2008). Basic theory of fractional differential equations. Elsevier, 69(8), 2677-2682.

[10] Podlubny, Igor (1998). Fractional differential equations: an introduction to fractional derivatives, fractional differential equations, to methods of their solution and some of their applications, Elsevier

[11] Lakshmikantham, V. (2017). Monotone Iterative Techniques for Discontinuous Nonlinear Differential Equations. Routledge.

[12] Dhage, Bapurao Ci(2013). Hybrid fixed point theory in partially ordered normed linear spaces and applications to fractional integral equations. Differ. Equ. Appl.5(2) 155-184.

[13] Dhage, Bapurao Chandrabahan (2014). Partially condensing mappings in partially ordered normed linar spaces and applications to functional integral equations. Tamkang Journal of Mathematics, 4(45),397-426.

[14] Dhage, BC (2015). Operator theoretic techniques in the theory of nonlinear hybrid differential equations. Nonlinear Anal. Forum, 20, 15-31.

[15] Dhage, Shyam B (2015). Approximating solutions of nonlinear first order ordinary differential equations. Global Journal of Mathematical Sciences (GJMS),2(2).

[16] Dhage, Bapurao C. and Dhage, Shyam B. (2014). Global attractivity and stability results for comparable solutions of nonlinear fractional integral equations. Nonlinear Studies, 2(21). 